\title{
Extralabyrinthine Manifestations of DFNA9
}

\author{
Andrew A. McCall ${ }^{1,2,5}$, Fred H. Linthicum Jr. ${ }^{3}$, Jennifer T. O’Malley ${ }^{1,2}$, Joe C. Adams ${ }^{1,2}$, \\ Saumil N. Merchant ${ }^{1,2}$, Marc K. Bassim ${ }^{4}$, Robert Gellibolian ${ }^{3}$, and Jose N. Fayad ${ }^{3,4}$, \\ ${ }^{1}$ Department of Otolaryngology, Massachusetts Eye and Ear Infirmary, 243 Charles Street, Boston, MA 02114, USA \\ ${ }^{2}$ Department of Otology and Laryngology, Harvard Medical School, Boston, MA 02114, USA \\ ${ }^{3}$ House Ear Institute, 256 South Lake Street, Los Angeles, CA 90057, USA \\ ${ }^{4}$ House Ear Clinic, Los Angeles, CA 90057, USA \\ ${ }^{5}$ Present address: Department of Otolaryngology, Eye and Ear Institute, University of Pittsburgh, 200 Lothrop Street Suite 500, \\ Pittsburgh, PA 15213, USA
}

Received: 14 May 2010; Accepted: 20 October 2010; Online publication: 4 November 2010

\begin{abstract}
DFNA9 is an autosomal dominant cause of nonsyndromic adult-onset sensorineural hearing loss with associated variable vestibular dysfunction caused by mutations in the COCH gene. DFNA9 has previously been characterized by the presence of unique histopathologic features limited to the cochlear and vestibular labyrinth. This report describes newly discovered extralabyrinthine findings within the middle ear in DFNA9 and discusses their implications. The histopathologic anatomy of extralabyrinthine structures was reviewed in 12 temporal bones from seven individuals with DFNA9 and compared with agematched controls. All temporal bones with DFNA9 had abnormal deposits within the tympanic membrane, incudomalleal joint, and incudostapedial joint. Hematoxylin and eosin stain and Movat's pentachrome stain both revealed different staining patterns of the extralabyrinthine deposits compared with the intralabyrinthine deposits suggesting that the composition of the deposits varies with location. The deposits within the tympanic membrane resembled cartilage morphologically and stained positively for aggrecan, an extracellular matrix protein found in cartilage. However, the cellular component of the tympanic membrane deposits did not stain with immunomarkers for chondrocytes (s100 and connective tissue
\end{abstract}

Correspondence to: Andrew A. McCall . Department of Otolaryngology . Eye and Ear Institute - University of Pittsburgh . 200 Lothrop Street Suite 500, Pittsburgh, PA 15213, USA. Telephone: +1-412-6472115; email: mccallaa@upmc.edu growth factor). These novel findings in DFNA9 have implications for the phenotypic expression of the disorder and the clinical workup of adult-onset sensorineural hearing loss.

Keywords: cochlin, $\mathrm{COCH}$, hearing loss, deafness

\section{INTRODUCTION}

Hereditary hearing loss is the most common sensory disorder of humans (Kochhar et al. 2007). Genetic hearing loss is divided into syndromic and non-syndromic types, and non-syndromic losses are further subdivided by inheritance pattern, which permits categorization of the heterogenous disorders that result in hearing loss. DFNA9 is a form of non-syndromic, autosomal dominantly inherited sensorineural hearing loss caused by mutations in the COCH gene encoding cochlin (Robertson et al. 1998). To date, 12 mutations within the COCH gene which result in DFNA9 have been described (Robertson et al. 2008). The clinical manifestations of DFNA9 include adult-onset progressive sensorineural hearing loss beginning between ages 20 and 50 with progression to anacusis, and vestibular dysfunction accompanying the hearing loss in some individuals (Robertson et al. 1998; Khetarpal 2000; Bischoff et al. 2005). The prevalence of DFNA9 is unknown as systematic genetic testing on the adultonset sensorineural hearing loss population has not 
been performed; however, the disease has been detected in multiple families across four continents (Robertson et al. 2006). Previous temporal bone histopathologic investigations in DFNA9 have revealed the presence of characteristic eosinophilic-staining deposits within the cochlear spiral ligament, spiral limbus, and spiral lamina as well as within the stroma underlying the utricular and saccular maculae and the cristae of the semicircular canals (Khetarpal et al. 1991; Merchant et al. 2000).

Prior histopathologic work on DFNA9 has focused on the pathologic changes within the inner ear. In a recent internal review of DFNA9 temporal bones, we discovered abnormalities of the middle ear that had not previously been characterized. The goal of the current study was to systematically study the extralabyrinthine structures of all temporal bones with DFNA9 at the Massachusetts Eye and Ear Infirmary and House Ear Institute. In the present study, we report new histopathologic findings located in the middle ear in DFNA9 and further characterize the nature of these new findings. The results have implications for the pattern of phenotypic expression of DFNA9 and for the clinical workup of adult-onset sensorineural hearing loss.

\section{METHODS}

\section{Morphological analysis of extralabyrinthine structures}

The human temporal bone collections at the Massachusetts Eye and Ear Infirmary and House Ear Institute were queried for subjects with DFNA9; 12 temporal bones from seven individuals were identified for study. Our study was approved by the Human Studies Committees at both institutions. All temporal bones were processed using the protocol outlined by Schuknecht (Schuknecht 1993). To summarize, temporal bones were removed at autopsy, placed in $10 \%$ formalin fixative, decalcified in ethylenediaminetetraacetate, embedded in celloidin, serially sectioned at a thickness of $20 \mu \mathrm{m}$, and every tenth section was stained with hematoxylin and eosin (H\&E). Sections were examined by light microscopy.

After confirming the histopathologic diagnosis of DFNA9 by identifying the characteristic eosinophilicstaining deposits within the cochlea and vestibular labyrinth, we systematically studied all extralabyrinthine structures of these bones. Special attention was paid to the mobile structures of the middle ear, including the tympanic membrane, incudostapedial joint, incudomalleal joint, stapediovestibular joint, anterior malleal ligament, tensor tympani tendon, and incudal ligament, after preliminary investigations showed abnormalities in several of these areas. Each DFNA9 temporal bone was compared with agematched controls without a history of otologic disease and without histopathologic evidence of otopathology except age-related change.

\section{Immunohistochemical and histochemical staining}

Immunohistochemical staining was performed to further characterize the newly discovered extralabyrinthine findings. Unstained sections adjacent to sections of interest identified by $\mathrm{H} \& \mathrm{E}$ were selected. Celloidin was removed prior to immunostaining with sodium methoxide as outlined in previous work (O'Malley et al. 2009). In short, sections were adhered to glass slides, a sodium methoxide mixture was applied to the celloidinembedded tissues and rinsed with pure methanol (this process was repeated twice), then the sections were immersed in descending methanol solutions, transferred to distilled water, and exposed to phosphate buffered saline. Immunostaining was then carried out as previously described (Adams 2002). In short, sections were blocked with $5 \%$ normal horse serum for $1 \mathrm{~h}$ and incubated overnight with primary antibody (Table 1). Serial dilutions were performed of each antibody to determine the optimal staining dilution. Biotinylated secondary antibodies appropriate for individual primary antibodies were placed for $1 \mathrm{~h}$, followed by avidin-biotin-horseradish peroxidase (Standard ABC kit, Vector Labs, Burlingame CA) for $1 \mathrm{~h}$. Sections were then colorized with $0.01 \%$ diaminobenzidine and $0.01 \%$ hydrogen peroxide for approximately $5 \mathrm{~min}$, washed in water, dehydrated, and coverslipped. A slight modification to the protocol was used to visualize aggrecan (biotinylated link and streptavidin-AP labels, DAKO, Carpenteria CA, followed by fast red application).

Histochemical staining techniques were also used to characterize the nature of the extralabyrinthine histopathologic changes. Movat's pentachrome stain was performed on unstained sections after celloidin was removed with clove oil as outlined in previous work (Sheehan and Hrapchak 1973; O'Malley et al. 2009). Sections were adhered to glass slides, dehydrated in ascending ethanol solutions, and trans-

TABLE 1

Primary antibody and concentration used to characterize the extralabyrinthine manifestations of DFNA9

\begin{tabular}{ll}
\hline Aggrecan & $1: 500$ \\
s100 & Undiluted \\
Connective tissue growth factor & $1: 1,000$ \\
\hline
\end{tabular}




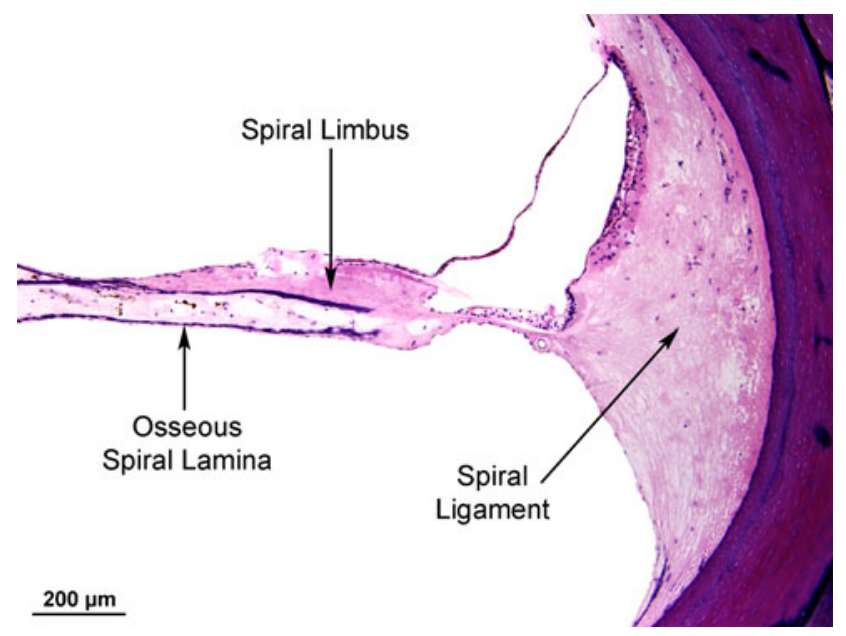

FIG. 1. Cochlear deposits in DFNA9. Note the eosinophilicstaining deposits within the spiral ligament and spiral limbus. Also note the hypocellularity of the spiral ligament and lack of dendritic processes from the cochlear nerve in the osseous spiral lamina. H\&E stain.

ferred to clove oil and left overnight. Sections were then hydrated to distilled water. Sections were placed in Alcian blue for $40 \mathrm{~min}$, in water for $3 \mathrm{~min}$, ethanol for $2 \mathrm{~h}$, water for $10 \mathrm{~min}$, rinsed with $70 \%$ ethanol, placed in resorchin-fuchsin for $20 \mathrm{~h}$, water for $10 \mathrm{~min}$, rinsed in distilled water, placed in Weigert's iron hematoxylin for $20 \mathrm{~min}$, water for $10 \mathrm{~min}$, placed in woodstain scarlet-acid fuchsin for $5 \mathrm{~min}$, rinsed with $0.5 \%$ acetic acid, placed under $5 \%$ phosphotungstic acid for $20 \mathrm{~min}$ and checked for color, rinsed with $0.5 \%$ acetic acid, washed with ethanol, placed in alcoholic saffron for 20 min, dehydrated, and coverslipped (Sheehan and Hrapchak 1973).
Appropriate age-matched controls were stained in parallel.

\section{RESULTS}

Twelve temporal bones were identified from seven individuals with a diagnosis of DFNA9. The group under study included three females and four males ranging in age from 45 to 86 . All 12 bones were confirmed to have the characteristic eosinophilic deposits within the cochlea and vestibular labyrinth that are pathognomonic for DFNA9 (Fig. 1). Prior genetic studies have identified the DFNA9 mutations responsible for the clinical and histopathologic findings in the temporal bones of five of the individuals studied (Table 2) (Robertson et al. 1998, 2006). The remaining two individuals share identical histopathologic features with the bones from individuals with specifically identified DFNA9 mutations. Twelve agematched control temporal bones were confirmed to be free of pathologic change (excepting age-related change).

\section{Histopathologic and immunohistochemical analysis}

The histopathologic extralabyrinthine manifestations of DFNA9 are summarized in Table 2. One striking finding within all DFNA9 temporal bones was the thickening of the middle layer of the tympanic membrane by deposits (Fig. 2). These tympanic membrane deposits occurred focally within the pars tensa and were of variable thickness, with the remain-

TABLE 2

\begin{tabular}{|c|c|c|c|c|c|c|c|c|c|c|c|c|}
\hline \multicolumn{13}{|c|}{ Extralabyrinthine histopathologic changes in DFNA9 by site } \\
\hline Case number & \multicolumn{2}{|l|}{1} & \multicolumn{2}{|l|}{2} & \multicolumn{2}{|l|}{3} & \multicolumn{2}{|l|}{4} & \multicolumn{2}{|l|}{5} & 6 & 7 \\
\hline Age and sex & \multicolumn{2}{|c|}{$86 F$} & \multicolumn{2}{|c|}{$67 F$} & \multicolumn{2}{|c|}{$59 F$} & \multicolumn{2}{|c|}{$82 \mathrm{M}$} & \multicolumn{2}{|c|}{$78 \mathrm{M}$} & $82 M$ & $45 M$ \\
\hline DFNA9 mutation & \multicolumn{2}{|c|}{$V 66 G$} & \multicolumn{2}{|c|}{ P51S } & \multicolumn{2}{|c|}{ V66G } & \multicolumn{2}{|c|}{ G88E } & & & \multicolumn{2}{|l|}{ G88E } \\
\hline Side & $L$ & $R$ & $L$ & $R$ & $L$ & $R$ & $L$ & $R$ & $L$ & $R$ & $L$ & $L$ \\
\hline Tympanic membrane & + & + & + & & + & + & + & + & + & & + & + \\
\hline Incudomalleal joint & + & + & + & & + & + & + & & + & + & + & + \\
\hline Incudostapedial joint & + & + & + & + & + & + & + & & + & + & + & + \\
\hline Stapediovestibular joint & - & - & - & - & - & - & - & - & - & - & - & - \\
\hline Anterior malleal ligament & - & - & - & & - & - & - & & + & - & - & - \\
\hline Tensor tympani tendon & - & - & - & & - & - & - & & - & - & + & - \\
\hline Incudal ligament & - & - & - & & - & - & - & & - & - & - & - \\
\hline Internal auditory canal & - & - & - & - & - & - & - & - & - & - & - & - \\
\hline
\end{tabular}

Each structure listed in Table 2 was examined for the presence of histopathologic findings in DFNA9. Specifically, abnormal findings (as indicated by a positive sign (+) above) were characterized by thickening of the structure under examination with disorganized material, often accompanied by the presence of an abnormal cellular component (see Figs. 2 and 5 for examples). If the structure was normal, it is indicated by a negative sign (-) above. Some temporal bones did not have all components preserved in a condition that would permit histopathological evaluation (cases $2 R, 4 R$, and $5 R$ ) - those structures which were unevaluable are indicated by a blank space in the table above. Specific DFNA9 mutations associated with each case have been included, when known. 

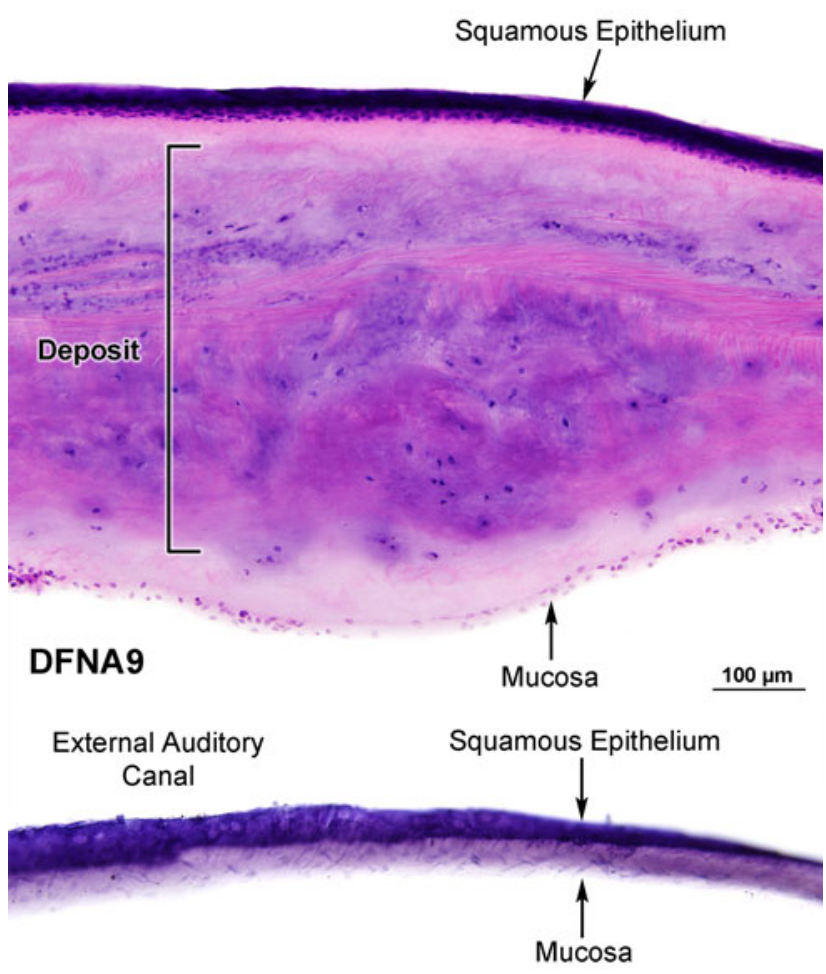

Middle

Ear

\section{CONTROL}

$100 \mu \mathrm{m}$

FIG. 2. Top, tympanic membrane deposits resembling cartilage in DFNA9. Note the mixed eosinophilic and basophilic staining material causing marked thickening of the tympanic membrane. The deposits are composed of cells surrounded by lacunae amongst disorganized whorls of extracellular material. Bottom, normal agematched control tympanic membrane at equal magnification. H\&E stain.

der of the tympanic membrane appearing normal. The proportion of the tympanic membrane within a given temporal bone affected with the deposits averaged $26 \%$ (range, $5 \%$ to $50 \%$ ). The staining characteristics of the deposits varied; the deposits typically consisted of a mixture of eosinophilic and basophilic staining patterns, however, on occasion, the deposits were almost entirely eosinophilic or basophilic. The morphologic appearance of the deposits resembled cartilage: cells were seen within lacunae and were surrounded by extracellular material. Immunostaining was performed to investigate the chondroid appearance of the deposits. The deposits stained positively with immunomarkers for aggrecan, a cartilaginous extracellular matrix protein (Fig. 3). However, the cellular component did not stain with chondrocyte markers s100 or connective tissue growth factor (CTGF) (Fig. 4).

The joints of the middle ear were also found to have histopathologic changes in DFNA9. In all DFNA9 temporal bones, the incudomalleal and incudostapedial joints were thickened with whorls of disorganized eosinophilic and basophilic stained material similar to that found within the tympanic membrane (Fig. 5). The entire joint space was affected in the incudomalleal and incudostapedial joints. For example, in the incudomalleal joint, the deposits filled the joint space from the medial to the lateral incudomalleal ligaments. Other mobile areas within the middle ear such as the stapediovestibular joint, tensor tympani tendon, anterior malleal ligament, and incudal ligament did not reliably demonstrate similar deposits in DFNA9. One individual demonstrated cartilage-like deposits within the tensor tympani tendon and another individual within the anterior malleal ligament; however, these findings were absent in the remainder of the specimens. Histopathologic evidence for confounding pathologies, such as otitis media and degenerative joint disease, was absent in all cases. Other extralabyrinthine areas of the temporal bones were not found to have any other histopathologic abnormalities.

None of the control specimens showed any abnormalities on light microscopy or immunostaining.

\section{Comparative evaluation of middle ear with inner ear deposits}

The deposits within the tympanic membrane and middle ear structures exhibited a variety of staining patterns, most commonly a mixture of eosinophilic and basophilic stains (Figs. 2 and 5), which differed from the uniform eosinophilic-staining characteristics of the deposits within the inner ear (Fig. 1) using a standard $\mathrm{H} \& \mathrm{E}$ stain. To confirm this apparent difference in the composition of the deposits, in light of its prior use in DFNA9, Movat's pentachrome stain was performed (Fig. 6) (Khetarpal et al. 1991). Movat's pentachrome is a histochemical staining technique, where color of staining represents the basic underlying composition of the tissue. For example, nuclei stain black, collagen yellow, ground substance blue, and fibrin red (Sheehan and Hrapchak 1973). The staining pattern within the inner ear confirmed the previously reported finding that the substance within the spiral ligament, spiral limbus, and stroma underlying the vestibular endorgans was ground substance/mucopolysaccharide (Khetarpal et al. 1991). The staining pattern of the deposits within the tympanic membrane and incudomalleal joints was different than that in the inner ear; the tympanic membrane 


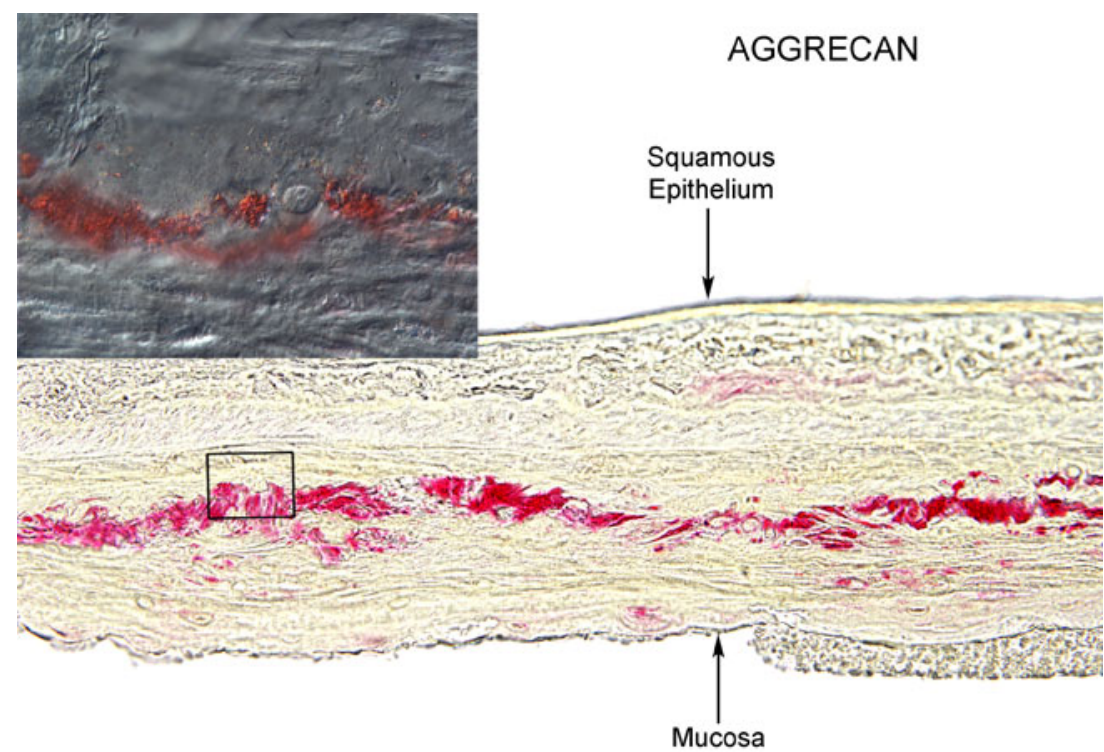

$100 \mu \mathrm{m}$ deposits appeared to be composed of a mixture of both ground substance and collagen stains, and the incudomalleal joints were composed of whorls of a peach color that perhaps represent a mixture of several substances. In an effort to further compare the middle ear and inner ear deposits, we attempted staining with anti-cochlin antibodies (graciously provided by Thomas Carey, Ph.D., Kresge Hearing Research Institute), but the results were inconclusive because of non-specific staining (data not shown).

\section{DISCUSSION}

DFNA9 has previously been characterized as a disorder solely afflicting the inner ear, resulting in sensorineural hearing loss and variable vestibular dysfunction. Prior investigations have shown the presence of inner ear deposits within the spiral ligament, spiral limbus, and the stroma underlying the vestibular sensory epithelium in DFNA9; cochlin has been detected by immunohistochemical staining in the normal and DFNA9 conditions in these locations (Khetarpal et al. 1991; Robertson et al. 2006). The present study demonstrates that the manifestations of DFNA9 are more widespread than previously thought. Specifically, the tympanic membranes in DFNA9 are characterized by the presence of focal deposits of extracellular material surrounding a cellular infiltrate resulting in a morphological appearance resembling cartilage. The incudomalleal and incudostapedial joints contain similar deposits. Because the morphologic appearance of the deposits within the tympanic membrane so
FIG. 3. Immunostaining for aggrecan, an extracellular component of cartilage. The extracellular component of the deposits within the tympanic membrane stains positively for aggrecan (red staining). Higher power view, taken using Nomarski optics, confirms that aggrecan localizes to an extracellular location (inset). closely resembled cartilage, we sought to further investigate the nature of these deposits. Cartilage is composed of chondrocytes surrounded by an extracellular matrix, and markers for each were selected for study. The immunohistochemical staining pattern of the deposits within the tympanic membrane are different from true cartilage. The cellular component did not stain positively for either s100 or CTGF, both of which stain chondrocytes. However, the extracellular component did stain positively for aggrecan, which is an extracellular component of cartilage. We conclude that the deposits within the tympanic membrane are cartilage-like, but are not representative of true cartilaginous metaplasia. The mechanism by which cartilage-like deposits develop within the middle ear is unknown. Although cochlin was initially discovered within the inner ear, it has since been reported to be widely expressed in multiple organs including the spleen, brain, kidneys, testis, uterus, intestine, and eye, among others (Robertson et al. 1994; Rodriguez et al. 2004; Picciani et al. 2007). The middle ear has yet to be specifically evaluated for cochlin expression. Diminished clearance of misfolded cochlin is believed to be the mechanism by which deafness associated with inner ear deposits in DFNA9 develop (Yao et al. 2010). Additionally the second von Willebrand domain within cochlin has been shown to interact with extracellular matrix proteins, collagen in particular (Nagy et al. 2008). We do not know if misfolded mutant cochlin also plays a role in the development of deposits within the middle ear of DFNA9 through interaction with extracellular matrix proteins; further study is warranted to specifically investigate this point. 
Tympanic Membrane - S100

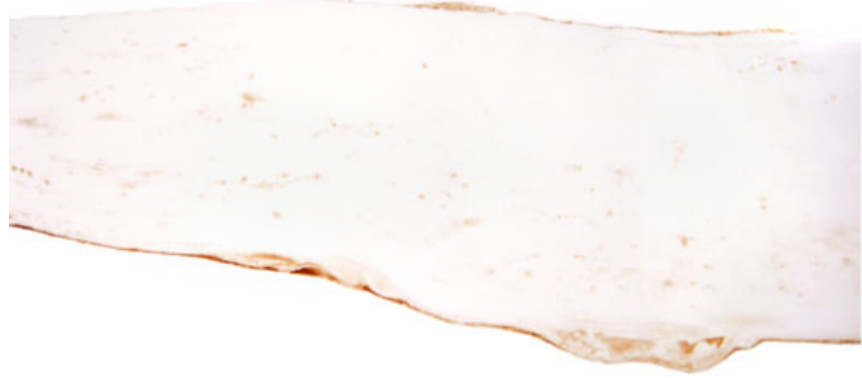

$200 \mu \mathrm{m}$

Tympanic Membrane - CTGF

$200 \mu \mathrm{m}$

FIG. 4. Chondrocyte immunohistochemical markers do not stain the cellular component of tympanic membrane deposits in DFNA9. The cellular component of the deposits in the tympanic membrane show little to no staining with either s100 (top, left) or CTGF (bottom, left). Contrast

Knowing that deposits are present both within the inner and middle ears of DFNA9, we sought to determine if the composition of the two was similar. We first evaluated standard H\&E sections. Deposits within the tympanic membrane consist of an amorphous and typically mixed eosinophilic and basophilic staining material surrounding cells in lacunae. Deposits within the incudomalleal and incudostapedial joints similarly are composed of deposits in a whorled pattern. In contrast, the deposits within the inner ear are uniformly eosinophilic and are associated with a relative hypocellularity. Movat's pentachrome staining provided further support for the apparent difference in composition of the middle ear and inner ear deposits. The inner ear deposits stained positively for mucopolysaccharides whereas the tympanic membrane deposits stained for a mixture of mucopolysaccharide and collagen stains surrounding nuclei. The incudomalleal joint stained with a

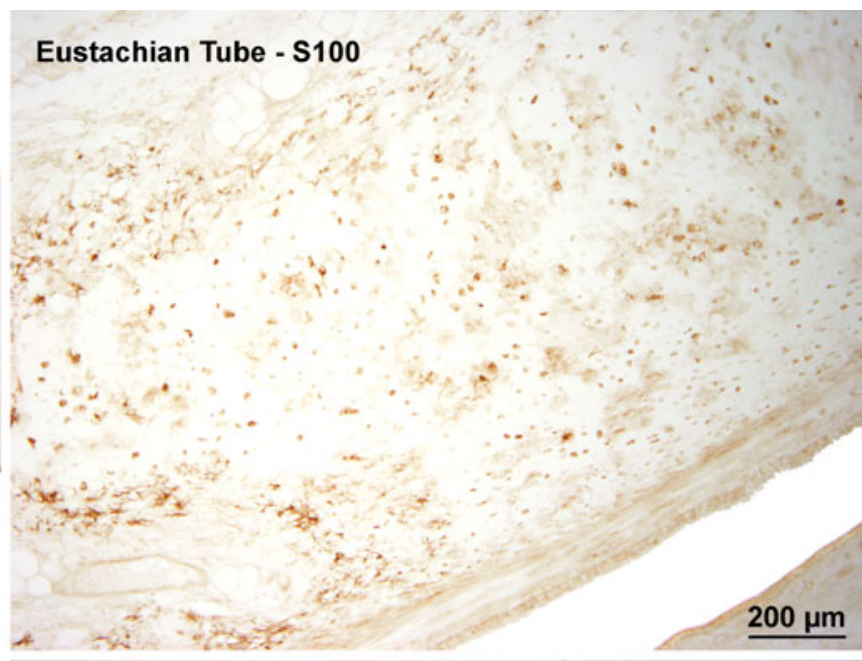

Eustachian Tube - CTGF

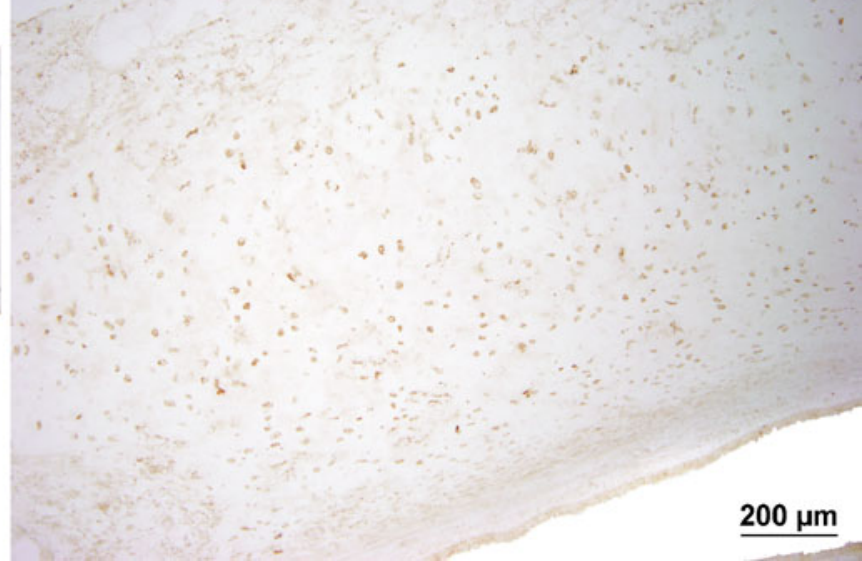

with the positive control staining of the eustachian tube, with extensive staining of chondrocytes with s100 (top, right) and CTGF (bottom, right). The images of the tympanic membrane deposits and cartilaginous eustachian tube are taken from within the same section for each stain.

different pattern as well. From the morphologic appearance and histochemical staining characteristics of these deposits, we conclude the deposits within the middle ear appear different than those within the inner ear. The reasons for the differential nature of the deposits between the middle ear and inner ear are unclear and further study is warranted to address this issue.

One salient fact discovered in the current study is that histopathologic deposits are present within both the middle and inner ears in DFNA9. A nonsyndromic genetic deafness with histopathologic findings within both the middle and inner ears is unique. DFN3, an X-linked form of non-syndromic hearing loss, does presumably affect the inner ear and middle ear as its clinical features include sensorineural hearing loss and stapes fixation, however, the histopathologic correlates of this disease have yet to be described (de Kok et al. 1995). At the current time, DFNA9 is the lone 


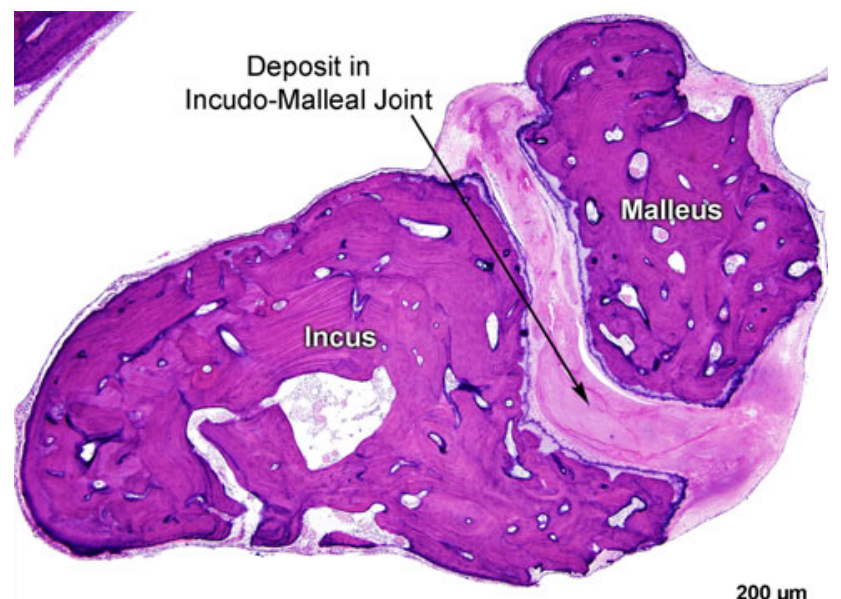

$200 \mu \mathrm{m}$

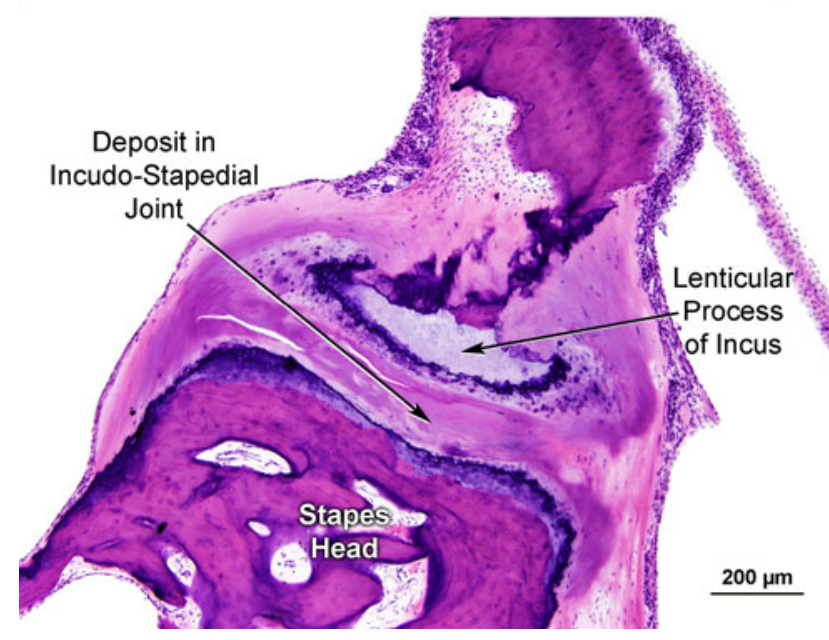

FIG. 5. Incudomalleal and incudostapedial joints show histopathologic changes similar to those seen in the tympanic membrane in DFNA9. The incudomalleal and incudostapedial joints are thickened with whorled material. H\&E stain.

form of non-syndromic genetic deafness with histopathologic change in both the middle and inner ears.

Hearing loss related to $\mathrm{COCH}$ gene mutations has been classified as a non-syndromic form of sensorineural hearing loss. There is mounting evidence to suggest that DFNA9 may ultimately represent a syndromic form of hearing loss. There is evidence that some families with DFNA9 have ophthalmological changes coincident with discovery of the hearing loss. In a study evaluating the ocular findings in DFNA9, three out of four DFNA9 families studied had vertical stria present on the cornea (Bischoff et al. 2007). It remains to be proven that the ocular findings are related to the $\mathrm{COCH}$ gene mutation rather than represent cosegregation with a nearby unrelated gene in these families. However, additional data exists that suggests cochlin may truly play a role in ocular disease. Proteomic studies have demonstrated differential expression of cochlin within the trabecular meshwork of glaucomatous patients
(Picciani et al. 2007; Picciani et al. 2009). These ophthalmologic findings along with evidence from this study showing histopathologic changes within the middle ear demonstrate that the effects of cochlin-related disease are not solely limited to the inner ear. Continued work investigating the distribution of $\mathrm{COCH}$ gene products and their physiologic activities along with studies carefully evaluating the clinical features of individuals with $\mathrm{COCH}$ gene mutations may provide evidence to further clarify the classification of DFNA9.

Another important question may be raised from the findings of the current study - does the disorder represent a pure sensorineural hearing loss or does a conductive component to the hearing loss exist given the fact that the deposits are found along the sound conduction pathway? We speculate that the middle ear deposits would likely have a negligible effect on sound transmission to the inner ear. The tympanic membrane deposits are focal and, analogous to the case of focal myringosclerosis, would likely not result in a conductive hearing loss (CayeThomasen et al. 2008). The deposits within the incudomalleal and incudostapedial joints may have a stiffening effect on these joints, which we would anticipate to have little, if any, detrimental clinical effect, and may even more efficiently transfer sound into the inner ear. Disorders of the ossicular chain which do result in conductive hearing loss typically result from anchoring the ossicular chain to another fixed structure (e.g., otosclerosis fixing the stapes to the bony labyrinth) or erosion and uncoupling of the ossicular chain (e.g., chronic otitis media eroding the incudostapedial joint) (Chole and McKenna 2001; Chien et al. 2009; Ebenezer and Rupa 2010). Supporting the assertion that the middle ear deposits do not contribute a conductive component to the hearing loss in DFNA9, prior clinical reports have shown isolated sensorineural hearing loss in affected family members in kindreds with DFNA9 (Halpin et al. 1996; Manolis et al. 1996). Detailed longitudinal audiometric studies should clarify the issue.

The presence of middle ear deposits in DFNA9 may be relevant to the clinical evaluation of families with adult-onset sensorineural hearing loss. We speculate that the deposits we have discovered histologically may be clinically detectable. First, the tympanic membrane deposits, especially when large, may be visible on otoscopic examination. Second, impedance measurements, such as tympanometry, may vary from normal, perhaps with a stiffened pattern. Third, imaging studies, such as computed tomography, may detect an area of thickening of the tympanic membrane corresponding to the deposits. If such diagnostic clues prove to be 

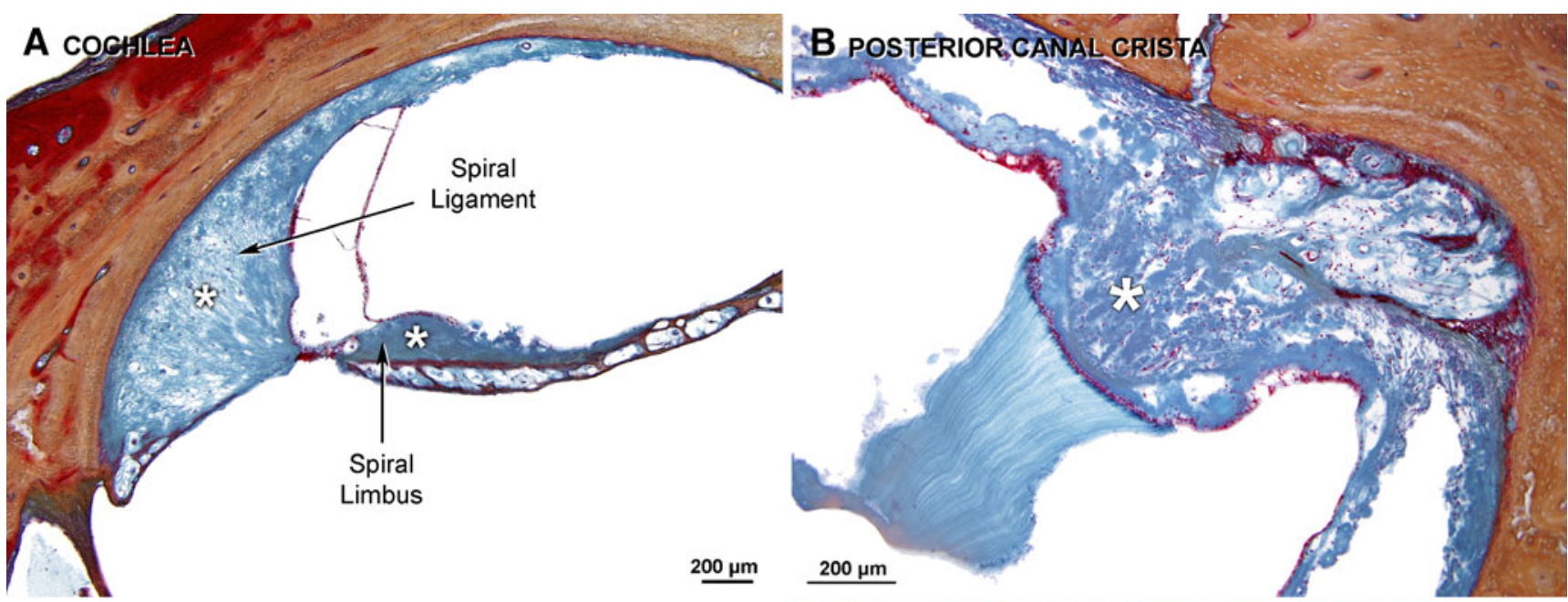

\section{TYMPANIC MEMBRANE}

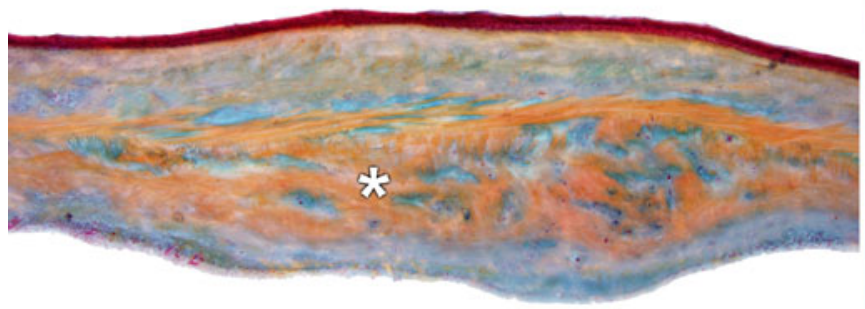

FIG. 6. Movat's pentachrome stain in DFNA9 confirms that the composition of the deposits in the middle ear differ from those in the inner ear. The spiral ligament and limbus stain blue (marked by asterisks in A) as does the stroma underlying the vestibular sensory epithelium (marked by asterisk in $B$ ) indicating the presence of ground substance/mucopolysaccharide. The tympanic membrane

present in clinical studies of DFNA9, these clinical tests would aid in the evaluation of individuals with adult-onset sensorineural hearing loss (especially in the setting of multiple hearing impaired family members) and could lead to appropriate genetic testing for $\mathrm{COCH}$ gene mutations.

\section{CONCLUSIONS}

The histopathologic manifestations of DFNA9 include changes within the middle and inner ears. The tympanic membrane in DFNA9 has focal deposits which are cartilage-like, and the incudomalleal and incudostapedial joints are thickened with deposits in a whorled pattern. The deposits within the middle ear appear to be different in composition than those in the inner ear. The phenotypic expression of $\mathrm{COCH}$

gene mutations in humans is more widespread than previously thought.

\section{ACKNOWLEDGEMENTS}

This work was supported by the National Institutes of Health (grants U24 DC 008559 and U24 DC 008625 Human Temporal Bone Consortium for Research Resource Enhancement of the National Institute on Deafness and Other Communication Disorders). We acknowledge Diane Jones and Barbara Burgess from the Massachusetts Eye and Ear Infirmary temporal bone histopathology laboratory for technical assistance with the project. We thank Andrew E. Rosenberg, MD bone and soft tissue pathology specialist from the Department of Pathology at the Massachusetts General Hospital for providing his expertise and guidance as well as providing the s100 antibody utilized in this project. 


\section{REFERENCES}

Adams JC (2002) Clinical implications of inflammatory cytokines in the cochlea: a technical note. Otol Neurotol 23:316-322

Bischoff AM, Huygen PL, Kemperman MH, Pennings RJ, Bom SJ, Verhagen WI, Admiraal RJ, Kremer H, Cremers CW (2005) Vestibular deterioration precedes hearing deterioration in the P51S COCH mutation (DFNA9): an analysis in 74 mutation carriers. Otol Neurotol 26:918-925

Bischoff AM, Pauw RJ, Huygen PL, Aandekerk AL, Kremer H, Cremers CW, Cruysberg JR (2007) Vertical corneal striae in families with autosomal dominant hearing loss: DFNA9/COCH. Am J Ophthalmol 143:847-852

Caye-Thomasen P, Stangerup SE, Jorgensen G, Drozdziewic D, Bonding P, Tos M (2008) Myringotomy versus ventilation tubes in secretory otitis media: eardrum pathology, hearing, and eustachian tube function 25 years after treatment. Otol Neurotol 29:649-657

Chien W, Northrop C, Levine S, Pilch BZ, Peake WT, Rosowski JJ, Merchant SN (2009) Anatomy of the distal incus in humans. J Assoc Res Otolaryngol 10:485-496

Chole RA, McKenna M (2001) Pathophysiology of otosclerosis. Otol Neurotol 22:249-257

de Kok YJ, van der Maarel SM, Bitner-Glindzicz M, Huber I, Monaco AP, Malcolm S, Pembrey ME, Ropers HH, Cremers FP (1995) Association between X-linked mixed deafness and mutations in the POU domain gene POU3F4. Science 267:685-688

EBENEZer J, RUPA V (2010) Preoperative predictors of incudal necrosis in chronic suppurative otitis media. Otolaryngol Head Neck Surg 142:415-420

Halpin C, Khetarpal U, McKenna M (1996) Autosomal-dominant progressive sensorineural hearing loss in a large North American family. Am J Audiol 5:105-111

Khetarpal U, Schuknecht HF, Gacek RR, Holmes LB (1991) Autosomal dominant sensorineural hearing loss. Pedigrees, audiologic findings, and temporal bone findings in two kindreds. Arch Otolaryngol Head Neck Surg 117:1032-1042

KHETARPAL U (2000) DFNA9 is a progressive audiovestibular dysfunction with a microfibrillar deposit in the inner ear. Laryngoscope 110:1379-1384

Kochhar A, Hildebrand MS, Smith RJ (2007) Clinical aspects of hereditary hearing loss. Genet Med 9:393-408

Manolis EN, Yandavi N, Nadol JB Jr, Eavey RD, McKenna M, Rosenbaum S, Khetarpal U, Halpin C, Merchant SN, Duyk GM, MacRae C, Seidman CE, Seidman JG (1996) A gene for non-syndromic autosomal dominant progressive postlingual sensorineural hearing loss maps to chromosome 14q12-13. Hum Mol Genet 5:1047-1050
Merchant SN, Linthicum FH, Nadol JB Jr (2000) Histopathology of the inner ear in DFNA9. Adv Otorhinolaryngol 56:212-217

Nagy I, Trexler M, Patthy L (2008) The second von Willebrand type A domain of cochlin has high affinity for type I, type II and type IV collagens. FEBS Lett 582:4003-4007

O’Malley JT, Burgess BJ, Jones DD, Adams JC, Merchant SN (2009) Techniques of celloidin removal from temporal bone sections. Ann Otol Rhinol Laryngol 118:435-441

Picciani R, Desai K, Guduric-Fuchs J, Cogliati T, Morton CC, Bhattacharya SK (2007) Cochlin in the eye: functional implications. Prog Retin Eye Res 26:453-469

Picciani RG, Diaz A, Lee RK, Bhattacharya SK (2009) Potential for transcriptional upregulation of cochlin in glaucomatous trabecular meshwork: a combinatorial bioinformatic and biochemical analytical approach. Invest Ophthalmol Vis Sci 50:3106-3111

Robertson NG, Khetarpal U, Gutierrez-Espeleta GA, Bieber FR, MoRTON CC (1994) Isolation of novel and known genes from a human fetal cochlear cDNA library using subtractive hybridization and differential screening. Genomics 23:42-50

Robertson NG, Lu L, Heller S, Merchant SN, Eavey RD, McKenna M, Nadol JB Jr, Mivamoto RT, Linthicum FH JR, Lubianca Neto JF, Hudspeth AJ, Seidman CE, Morton CC, Seidman JG (1998) Mutations in a novel cochlear gene cause DFNA9, a human nonsyndromic deafness with vestibular dysfunction. Nat Genet 20:299-303

Robertson NG, Cremers CW, Huygen PL, Ikezono T, Krastins B, Kremer H, Kuo SF, Liberman mC, Merchant SN, Miller CE, Nadol JB Jr, Sarracino DA, Verhagen WI, Morton CC (2006) Cochlin immunostaining of inner ear pathologic deposits and proteomic analysis in DFNA9 deafness and vestibular dysfunction. Hum Mol Genet 15:1071-1085

Robertson NG, Jones SM, Sivakumaran TA, Giersch AB, Jurado SA, Call LM, Miller CE, Maison SF, Liberman MC, Morton CC (2008) A targeted Coch missense mutation: a knock-in mouse model for DFNA9 late-onset hearing loss and vestibular dysfunction. Hum Mol Genet 17:3426-3434

Rodriguez CI, Cheng JG, Liu L, Stewart CL (2004) Cochlin, a secreted von Willebrand factor type a domain-containing factor, is regulated by leukemia inhibitory factor in the uterus at the time of embryo implantation. Endocrinology 145:1410-1418

Schuknecht HF (1993) Pathology of the ear, 2nd edn. Lea and Febiger, Philadelphia

Sheehan DC, Hrapchak BB (1973) Theory and practice of histotechnology. C. V. Mosby Co, Saint Louis

Yao J, Py BF, Zhu H, BaO J, YUAN J (2010) Role of protein misfolding in DFNA9 hearing loss. J Biol Chem 285:14909-14919 